Laser Chem. 1987, Vol. 7, pp. 197-211

Photocopying permitted by license only

(C) 1987 Harwood Academic Publishers GmbH

Printed in the United Kingdom

\title{
Internal Rotation of the Methyl Group in the Electronically Excited State: $o$ - and $m$-Toluidine
}

\author{
KATSUHIKO OKUYAMA, NAOHIKO MIKAMI and MITSUO ITO† \\ Department of Chemistry, Faculty of Science, Tohoku University, Sendai \\ 980, Japan
}

The fluorescence excitation and dispersed fluorescence spectra of jet-cooled $o$ - and $m$-toluidine were observed. Vibrational analysis of the spectra provided us with the potentials for the internal rotation of the $\mathrm{CH}_{3}$ group in both ground and excited states. In $o$-toluidine, a large potential barrier to the internal rotation in the ground state is practically removed in the excited state. On the other hand, a nearly free internal rotation of the $\mathrm{CH}_{3}$ group in the ground state of $m$-toluidine gains a large barrier by the electronic excitation. The great change in the barrier height upon the electronic excitation is more remarkable than that found for fluorotoluene. A close relationship between the barrier height and the $\pi$ electron density at the ring carbon atom was found, indicating the hyperconjugation as the origin of the barrier height in the absence of steric hindrance.

KEY WORDS: Internal rotation; methyl group; toluidine; laser spectroscopy; supersonic jet; hyperconjugation.

\section{INTRODUCTION}

Recently, large amplitude motions of molecules have attracted a great deal of interest with respect to their vibrational potentials in electronically ground and excited states ${ }^{1-4}$ and their role in various relaxation processes ${ }^{5,6}$. In a series of our studies on large-amplitude motion of large polyatomic molecules, it was demonstrated that electronic

† To whom correspondence should be addressed. 
spectra of molecules in supersonic jets obtained by the use of various laser spectroscopies are very useful for the determination of the potentials $^{7-9}$ and for the elucidation of the dynamical behaviors ${ }^{10-11}$ of these molecules. One of the achievements of the studies is the finding of a great change in the potential upon electronic excitation. In our previous study on $o-, m$ - and $p$-fluorotoluene ${ }^{8}$, we showed that a large barrier to the internal rotation of the $\mathrm{CH}_{3}$ group in the ground state of $o$-fluorobenzene is almost removed in the lowest excited $S_{1}\left(\pi, \pi^{*}\right)$ state. On the other hand, a nearly free internal rotation of the $\mathrm{CH}_{3}$ group in the ground state of $m$ - or $p$-fluorotoluene is hindered in the $S_{1}$ state and this is especially marked in $m$-fluorotoluene. The results indicated that the internal rotation of a $\mathrm{CH}_{3}$ group is very sensitive to the nature of the electronic state and also to the location of the $\mathrm{CH}_{3}$ group attached to the parent molecule. $\mathrm{A} \mathrm{CH}_{3}$ group generally only weakly perturbs the electronic structure of a parent molecule. The internal rotation of a $\mathrm{CH}_{3}$ group, however, is expected to be greatly affected by the change in the electronic environs of the $\mathrm{CH}_{3}$ group. Therefore, the internal rotation provides us with a good measure for characterization of the electronic states of a molecule.

The present study is an extension of our previous study on fluorotoluene to $o$ - and $m$ - toluidine which have an $\mathrm{NH}_{2}$ group instead of the fluorine atom of fluorotoluene. We observed the fluorescence excitation and dispersed fluorescence spectra of jet-cooled $o$ - and $m$ toluidine due to the $S_{1}\left(\pi, \pi^{*}\right)-S_{0}$ transition. Many low frequency vibronic bands associated with the internal rotational levels in the $S_{0}$ and $S_{1}$ states were observed. From the analysis of the vibronic bands, the potentials for the internal rotation of the $\mathrm{CH}_{3}$ group were accurately determined for both the $S_{0}$ and the $S_{1}$ states. The results obtained are parallel to those of $o$ - and $m$-fluorotoluene, i.e. a large barrier to the internal rotation of $o$-toluidine in its $S_{0}$ state almost disappears in the $S_{1}$ state, while in $m$-toluidine an almost free internal rotation in the $S_{0}$ state gains a great barrier in the $S_{1}$ state. The great change in the barrier height upon electronic excitation is more remarkable than that in fluorotoluene. The barrier height for $m$-fluorotoluene and $m$ toluidine, together with that for $m$-cresol (which will be reported in detail elsewhere), show a clear relationship between the barrier height and the $\pi$ electron density of the meta ring carbon atom of the parent molecule to which the $\mathrm{CH}_{3}$ group is attached. The relationship strongly suggests that the barrier to internal rotation arises from hyperconju- 
gation. A possible cause for the disappearance of the barrier by the electronic excitation in $o$-toluidine is also discussed.

\section{EXPERIMENTAL}

The pulsed supersonic free-jet apparatus has already been described elsewhere ${ }^{12}$. The sample was heated to $330 \mathrm{~K}$ in a nozzle chamber to obtain sufficient vapor pressure and seeded in helium carrier gas. The gaseous mixture $(\sim 5 \mathrm{~atm}$.) was expanded into a vacuum chamber $\left(\sim 10^{-5}\right.$ Torr $)$ through an orifice of $0.8 \mathrm{~mm}$ diameter. The fluorescence excitation spectra were obtained by monitoring the total fluorescence with a photomultiplier (HTV R-562). The photocurrent was averaged by a boxcar integrator (Brook deal 9415/9425) and recorded. The exciting light used was the second harmonic of a dye laser (Molectron DL-14P) pumped by a nitrogen laser (Molectron UV-24). The laser resolution was about $1.0 \mathrm{~cm}^{-1}(\mathrm{fwhm})$. The dispersed fluorescence spectra were measured by a Nalumi $0.75-\mathrm{m}$ monochromator with a $0.04-\mathrm{mm}$ slit width and the spectral resolution was about $6 \mathrm{~cm}^{-1}$ (fwhm). The signal was detected by a photomultiplier (HTV R-928) and recorded by the same integrator system as that used for the fluorescence excitation spectra.

$o$-Toluidine and $m$-toluidine were purchased from Tokyo Kasei and purified by distillation.

\section{RESULTS AND DISCUSSION}

\section{Fluorescence excitation and dispersed fluorescence spectra}

Figure 1 shows the fluorescence excitation spectrum of jet-cooled $o$-toluidine due to the $S_{1}\left(\pi, \pi^{*}\right) \leftarrow S_{0}$ transition. The spectrum exhibits a well-resolved vibrational structure. There exist many low frequency bands on the higher frequency side of the lowest frequency band at $34316 \mathrm{~cm}^{-1}$. The main bands are those shifted by $3,28,58,91$, 142 and $197 \mathrm{~cm}^{-1}$ from the $34316 \mathrm{~cm}^{-1}$ band. They are connected by a line in the figure. Similar band structures are seen all over the spectrum, some of them being shown in the figure. These low frequency bands are very similar in structure to those observed in $o$-fluorotoluene reported in a previous study ${ }^{8}$, the latter being assigned to the transitions between the internal rotational levels of the $\mathrm{CH}_{3}$ 


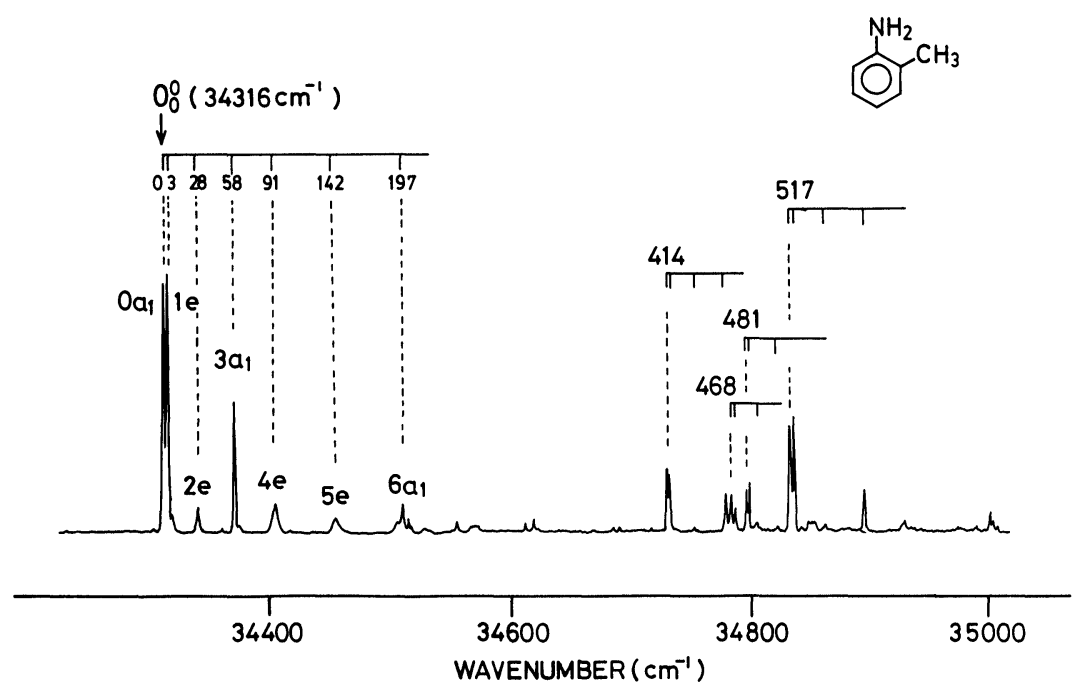

Figure 1 Fluorescence excitation spectrum of jet-cooled $o$-toluidine. Bands associated with internal rotational levels of the methyl group in $S_{1}$ are indicated. Frequencies measured from the 0,0 band are shown.

group in the $S_{0}$ and $S_{1}$ states. The similarity suggests the assignments of the low frequency bands of $o$-toluidine in parallel to those given for $o$-fluorotoluene and these assignments are given in the figure. The internal rotational levels in the $S_{1}$ state are denoted by a combination of the rotational quantum number, $m$, of a one-dimensional free rotor and the symmetry species of the permutation inversion group isomorphous to $C_{3 v}$ point group ${ }^{13,14}$. The assignments are supported from the dispersed fluorescence spectra which will be described below.

Figure 2a shows part of the dispersed fluorescence spectrum of jet-cooled $o$-toluidine obtained by excitation of the $34316 \mathrm{~cm}^{-1}\left(0 a_{1}\right)$ band in the excitation spectrum. It is a difficult task to find the bands associated with the ground state internal rotational levels among many bands. However, when exciting the $3 a_{1}\left(0+58 \mathrm{~cm}^{-1}\right)$ and $6 a_{1}(0+$ $197 \mathrm{~cm}^{-1}$ ) bands in the excitation spectrum, bands at 361 and $578 \mathrm{~cm}^{-1}$ show intensity enhancement in the dispersed fluorescence spectra as shown in Figure 2(b and c). Taking into account the $a_{1} \longleftrightarrow a_{1}$ selection rule for the transition between the internal rotational levels, the bands at 361 and $578 \mathrm{~cm}^{-1}$ can be assigned to $3 a_{1}$ and $6 a_{1}$ levels, respectively, in the $S_{0}$ state. 
(a) $\underset{0 a_{1}}{0}$ exc.

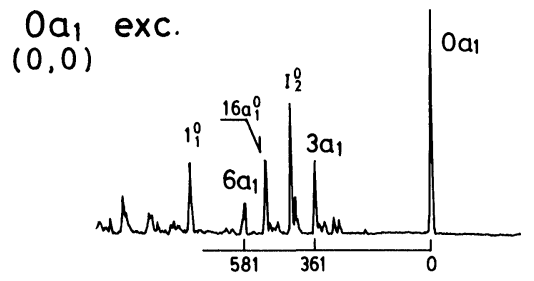

(b) $3 a_{1}$ exc.

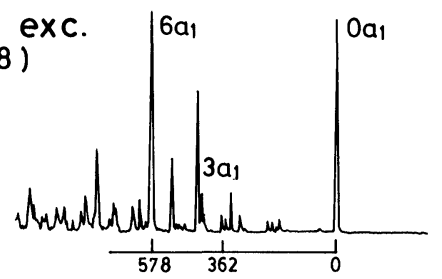

(c) $6 a_{1}$ exc.

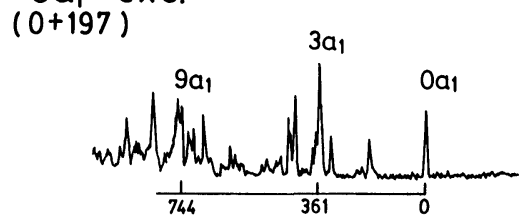

Figure 2 Dispersed fluorescence spectra of jet-cooled $o$-toluidine obtained by exciting the $a_{1}$ bands in Figure 1. Each spectrum is shown by relative frequency measured from the excitation frequency.

Figure 3 shows the dispersed fluorescence spectra obtained by exciting the $1 e\left(0+3 \mathrm{~cm}^{-1}\right), 2 e\left(0+28 \mathrm{~cm}^{-1}\right), 4 e\left(0+91 \mathrm{~cm}^{-1}\right)$ and $5 e$ $\left(0+142 \mathrm{~cm}^{-1}\right)$ bands in the excitation spectrum. It is seen from the figure that the bands at $0,182,363,472,587$ and $683 \mathrm{~cm}^{-1}$ show characteristic intensity enhancements in the individual dispersed fluorescence spectra. Therefore, they can be assigned to $1 e, 2 e 4 e, 5 e, 7 e$ and $8 e$ levels in the $S_{0}$ state because of the $e \longleftrightarrow e$ selection rule. Thus, we obtained the energies of the internal rotational levels in both the $S_{0}$ and $S_{1}$ states, which are listed in Table I. In the $S_{0}$ state, the $3 a_{1}$ and $4 e$ levels are nearly degenerate in energy and the frequency difference between the $6 a_{1}$ and $7 e$ levels is about $10 \mathrm{~cm}^{-1}$. This suggests a large potential barrier in the $S_{0}$ state compared with that in the $S_{1}$ state. The above assignments will be further confirmed from the calculated potentials and relative intensity distributions of the internal rotational bands in the fluorescence excitation and dispersed fluorescence spectra, which will be described later. 


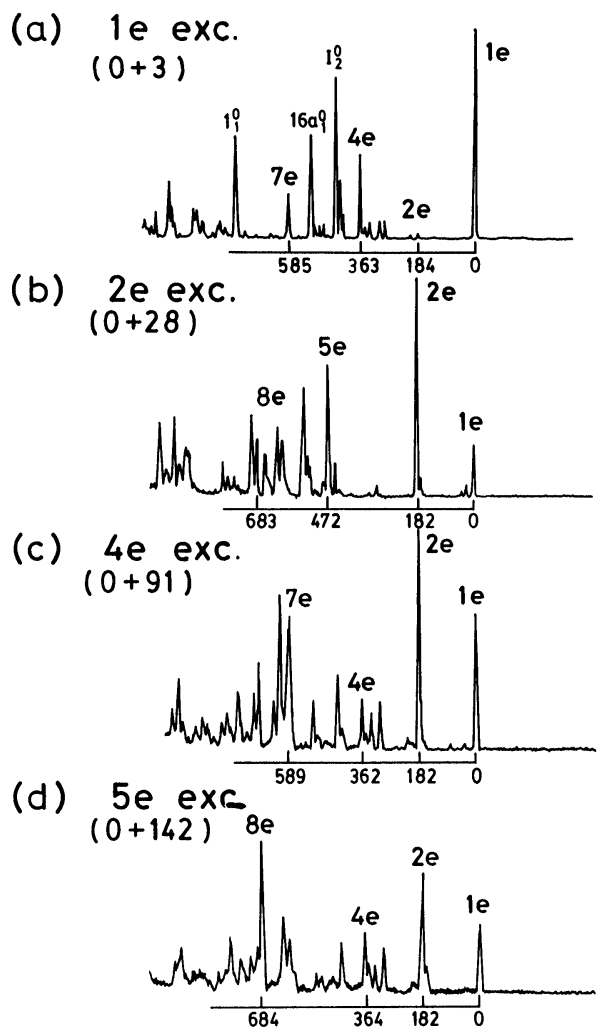

Figure 3 Dispersed fluorescence spectra of jet-cooled $o$-toluidine obtained by exciting the $e$ bands in Figure 1. Each spectrum is shown by relative frequency measured from the excitation frequency.

In Figure 4 is shown the fluorescence excitation spectrum of jetcooled $m$-toluidine due to the $S_{1}\left(\pi, \pi^{*}\right) \leftarrow S_{0}$ transition. The structure of low frequency bands less than $400 \mathrm{~cm}^{-1}$ is again similar to that of $m$-fluorotoluene reported in a previous paper $^{8}$, although some of the bands accidentally coincide in energy. The assignments of the low frequency bands can be made in parallel to those for $\mathrm{m}$ fluorotoluene and they are shown in the figure. A band at $33818 \mathrm{~cm}^{-1}$ $\left(0 a_{1}\right)$ is the $0_{0}^{0}$ band. A band red-shifted by $5.0 \mathrm{~cm}^{-1}$ from the $0_{0}^{0}$ band is the hot band due to the transition from the $1 e$ level in $S_{0}$ to the $1 e$ level 
Table I Internal rotational levels of $o$-toluidine in ground and excited states.

\begin{tabular}{|c|c|c|c|c|}
\hline \multirow{2}{*}{$\begin{array}{l}\text { Level } \\
\text { species }\left(C_{3 v}\right)\end{array}$} & \multicolumn{2}{|c|}{ Ground state $\left(\mathrm{cm}^{-1}\right)$} & \multicolumn{2}{|c|}{ Excited state $\left(\mathrm{cm}^{-1}\right)$} \\
\hline & Observed & Calculated $^{\mathrm{a}}$ & Observed & Calculated $^{\mathrm{b}}$ \\
\hline $0 a_{1}$ & 0 & 0 & 0 & 0 \\
\hline $1 e$ & 0 & 0 & 3 & 4 \\
\hline $2 e$ & 182 & 190 & 28 & 28 \\
\hline $3 a_{2}$ & - & 190 & 47 & 49 \\
\hline $3 a_{1}$ & 361 & 356 & 58 & 58 \\
\hline $4 e$ & 363 & 356 & 91 & 91 \\
\hline $5 e$ & 472 & 491 & 142 & 139 \\
\hline $6 a_{2}$ & - & 497 & - & 198 \\
\hline $6 a_{1}$ & 578 & 579 & 197 & 198 \\
\hline $7 e$ & 587 & 604 & - & 268 \\
\hline $8 e$ & 683 & 670 & - & 349 \\
\hline $9 a_{2}$ & - & 745 & - & 441 \\
\hline $9 a_{1}$ & 744 & 749 & - & 438 \\
\hline
\end{tabular}

a Calculated with $V_{3}=703 \mathrm{~cm}^{-1}, V_{6}=62 \mathrm{~cm}^{-1}$ and $B=5.28 \mathrm{~cm}^{-1}$.

b Calculated with $V_{3}=40 \mathrm{~cm}^{-1}, V_{6}=-11 \mathrm{~cm}^{-1}$ and $B=5.40 \mathrm{~cm}^{-1}$.

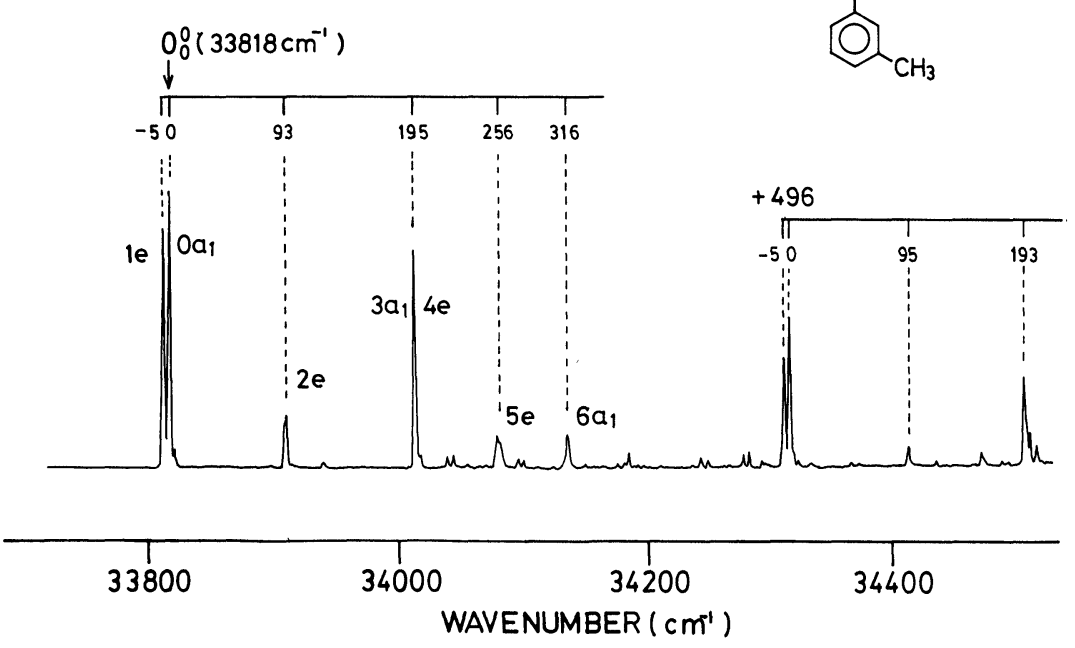

Figure 4 Fluorescence excitation spectrum of jet-cooled $m$-toluidine. Band associated with internal rotational levels of the methyl group in $S_{1}$ are indicated. Frequencies measured from the 0,0 band are shown. 
in $S_{1}$. The appearance of this band on the lower frequency side of the $0_{0}^{0}$ band suggests that the barrier to the internal rotation is higher in the $S_{1}$ state than in the $S_{0}$ state. The assignments of the low frequency bands in the fluorescence excitation spectrum are confirmed from the observation of the dispersed fluorescence spectra obtained by excitation of the low frequency bands, which are shown in Figure 5. As is seen from the figure, in the dispersed fluorescence spectra obtained by exciting the bands of $a_{1}$ species in the excitation spectrum, bands at 51 and $192 \mathrm{~cm}^{-1}$ measured from the exciting band commonly appear, while another set of bands at 17,81 and $130 \mathrm{~cm}^{-1}$ are commonly observed in the $e$ level dispersed fluorescence spectra. From the $a_{1} \longleftrightarrow a_{1}$ and $e \longleftrightarrow e$ selection rules, the former set of the bands can be assigned to the $a_{1}$ levels in the $S_{0}$ state and the latter set to the $e$ levels. The energies of the internal rotational levels in both the $S_{0}$ and $S_{1}$ states thus obtained are listed in Table II. The assignments will be further confirmed later by the calculated energy levels and intensity distributions of the internal rotational bands.
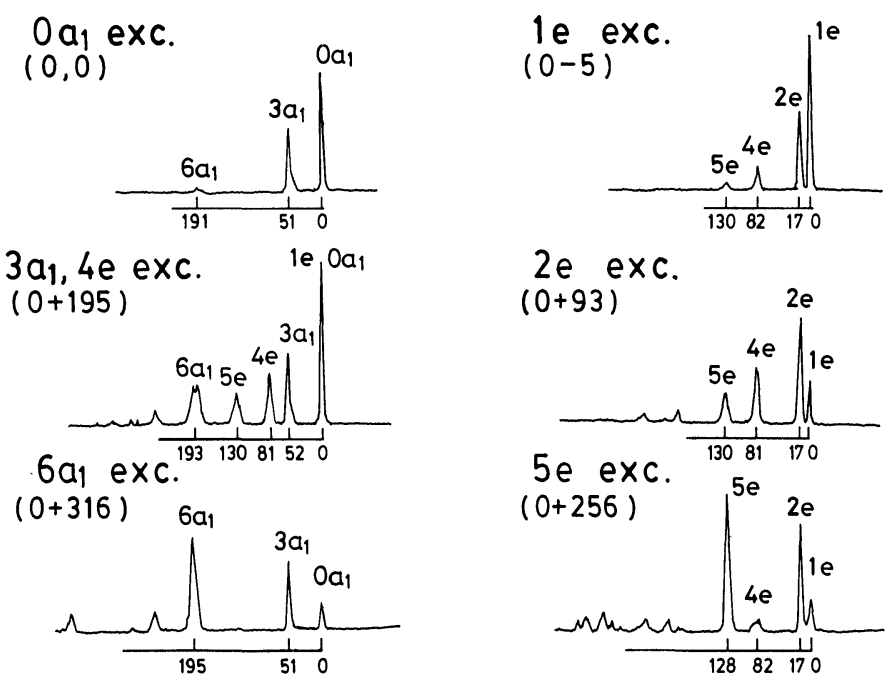

$2 e$ exc.

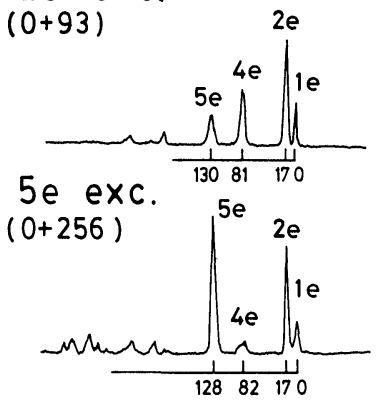

Figure 5 Dispersed fluorescence spectra of jet-cooled $m$-toluidine obtained by exciting the bands in Figure 4. Each spectrum is shown by relative frequency measured from the excitation frequency. 
Table II Internal rotational levels of $m$-toluidine in ground and excited states.

\begin{tabular}{|c|c|c|c|c|}
\hline \multirow{2}{*}{$\begin{array}{l}\text { Level } \\
\text { species }\left(C_{3 v}\right)\end{array}$} & \multicolumn{2}{|c|}{ Ground state $\left(\mathrm{cm}^{-1}\right)$} & \multicolumn{2}{|c|}{ Excited state $\left(\mathrm{cm}^{-1}\right)$} \\
\hline & Observed & Calculated $^{\mathrm{a}}$ & Observed & Calculated $^{\mathrm{b}}$ \\
\hline $0 a_{1}$ & 0 & 0 & 0 & 0 \\
\hline $1 e$ & 0 & 0 & -5 & -5 \\
\hline $2 e$ & 17 & 17 & 93 & 97 \\
\hline $3 a_{2}$ & - & 46 & - & 102 \\
\hline $3 a_{1}$ & 51 & 51 & 195 & 189 \\
\hline $4 e$ & 82 & 81 & 195 & 191 \\
\hline $5 e$ & 130 & 129 & 256 & 255 \\
\hline $6 a_{2}$ & - & 194 & - & 295 \\
\hline $6 a_{1}$ & 192 & 194 & 316 & 314 \\
\hline $7 e$ & 252 & 258 & - & 362 \\
\hline $8 e$ & - & 339 & - & 437 \\
\hline
\end{tabular}

a Calculated with $V_{3}=9 \mathrm{~cm}^{-1}, V_{6}=-10 \mathrm{~cm}^{-1}$ and $B=5.37 \mathrm{~cm}^{-1}$.

${ }^{b}$ Calculated with $V_{3}=317 \mathrm{~cm}^{-1}, V_{6}=-19 \mathrm{~cm}^{-1}$ and $B=5.24 \mathrm{~cm}^{-1}$.

\section{Potentials for internal rotation in $\mathbf{S}_{\mathbf{0}}$ and $\mathbf{S}_{\mathbf{1}}$ states}

The energy level structures for the internal rotation of the $\mathrm{CH}_{3}$ group in the $S_{0}$ and $S_{1}$ states for $o$ - and $m$-toluidine have now been obtained. If we assume that the methyl top and the benzene framework are rigid rotors, the wave function $\psi(\varphi)$ for the internal rotation should satisfy the following equation

$$
\left[-B \frac{\mathrm{d}^{2}}{\mathrm{~d} \varphi^{2}}+V(\varphi)\right] \psi(\varphi)=E \psi(\varphi)
$$

where $\varphi$ is the torsional angle between the two rotors and $B$ is the reduced rotational constants of the rotors about the methyl top axis. The potential $V(\varphi)$ is assumed by

$$
V(\varphi)=\frac{V_{3}}{2}(1-\cos 3 \varphi)+\frac{V_{6}}{2}(1-\cos 6 \varphi)
$$

Equation (1) can be solved by expanding $\psi(\varphi)$ by a basis set of one-dimensional free rotor wave functions and by diagonalizing the Hamiltonian matrix ${ }^{5}$. Three unknown parameters $B, V_{3}$ and $V_{6}$ were determined by adjusting them to give the calculated energies which give a best fit to the observed energies. The relative intensity distributions of the bands due to the internal rotation in the fluorescence excitation and dispersed fluorescence spectra provide us with a useful 
check of the potentials and also with the relative relation between the $S_{0}$ and $S_{1}$ potentials with respect to the torsional angle. The procedure for the calculation of the intensity distribution was described in detail in a previous paper ${ }^{8}$.

In Table III are summarized the potential parameters $V_{3}$ and $V_{6}$ and the reduced rotational constant $B$ obtained from the above calculations. Figures 6 and 7 show the potentials and energy levels in the $S_{0}$ and $S_{1}$ states. Comparison between the calculated and observed level energies is given in Tables I and II. It is seen that the agreement between the observed and calculated energies is satisfactory. In Figures 8 and 9 are shown comparisons between the observed and calculated intensity distributions of the fluorescence excitation and dispersed fluorescence spectra. The agreement is generally very good when assuming the same stable conformations of the $\mathrm{CH}_{3}$ group for both the $S_{0}$ and the $S_{1}$ states. In the case of $o$-fluorotoluene, we found the conformation change upon electronic excitation. However, in $o$-toluidine, no sign of the conformation change was found.

It is concluded from the above results that in $o$-toluidine, the barrier to the internal rotation is as large as $700 \mathrm{~cm}^{-1}$ in the $S_{0}$ state, but it dramatically decreases to only $40 \mathrm{~cm}^{-1}$ in the $S_{1}$ state. A completely reversed relationship is found in $m$-toluidine, where the barrier is very small $\left(9 \mathrm{~cm}^{-1}\right)$ in the $S_{0}$ state, but increases to as large as $300 \mathrm{~cm}^{-1}$ in the $S_{1}$ state. The decrease of the barrier height in the $o$-isomer and the increase in the $m$-isomer upon electronic excitation are the same as those for $o$ - and $m$-fluorotoluene. However, the effect upon the electronic excitation is about three times more remarkable in toluidine than in fluorotoluene.

We shall first consider the ground-state potential. Toluidine has two flexible groups of $\mathrm{NH}_{2}$ and $\mathrm{CH}_{3}$. It is well known that the parent Table III Potential parameters and rotational constants for internal rotation of $\mathrm{CH}_{3}$ in ground and excited states.

\begin{tabular}{|c|c|c|}
\hline & Ground state $\left(\mathrm{cm}^{-1}\right)$ & Excited state $\left(\mathrm{cm}^{-1}\right)$ \\
\hline$o$-Toluidine & $\begin{aligned} V_{3} & =703 \\
V_{6} & =62 \\
B & =5.28\end{aligned}$ & $\begin{aligned} V_{3} & =40 \\
V_{6} & =-11 \\
B & =5.40\end{aligned}$ \\
\hline$m$-Toluidine & $\begin{aligned} V_{3} & =9 \\
V_{6} & =-10 \\
B & =5.37\end{aligned}$ & $\begin{aligned} V_{3} & =317 \\
V_{6} & =-19 \\
B & =5.24\end{aligned}$ \\
\hline
\end{tabular}


molecule-aniline-has the inversion vibration of $\mathrm{NH}_{2}$ group analogous to the "umbrella" vibration of ammonia. The potential barrier opposing coplanarity of the $\mathrm{NH}_{2}$ group and benzene ring is known to be $526 \mathrm{~cm}^{-116}$. The inversion barrier height is 558 and $528 \mathrm{~cm}^{-1}$, respectively, in $o$ - and $m$-toluidine ${ }^{17}$. Therefore, the substitution of an aromatic hydrogen atom by a $\mathrm{CH}_{3}$ group does not greatly affect the inversion potential. In contrast to the insensitivity of the inversion potential, the potential for the internal rotation of the $\mathrm{CH}_{3}$ group changes greatly depending upon its location. These facts show that the electronic structure of toluidine is essentially determined by that of the parent molecule (aniline) and the $\mathrm{CH}_{3}$ group is electronically a weak

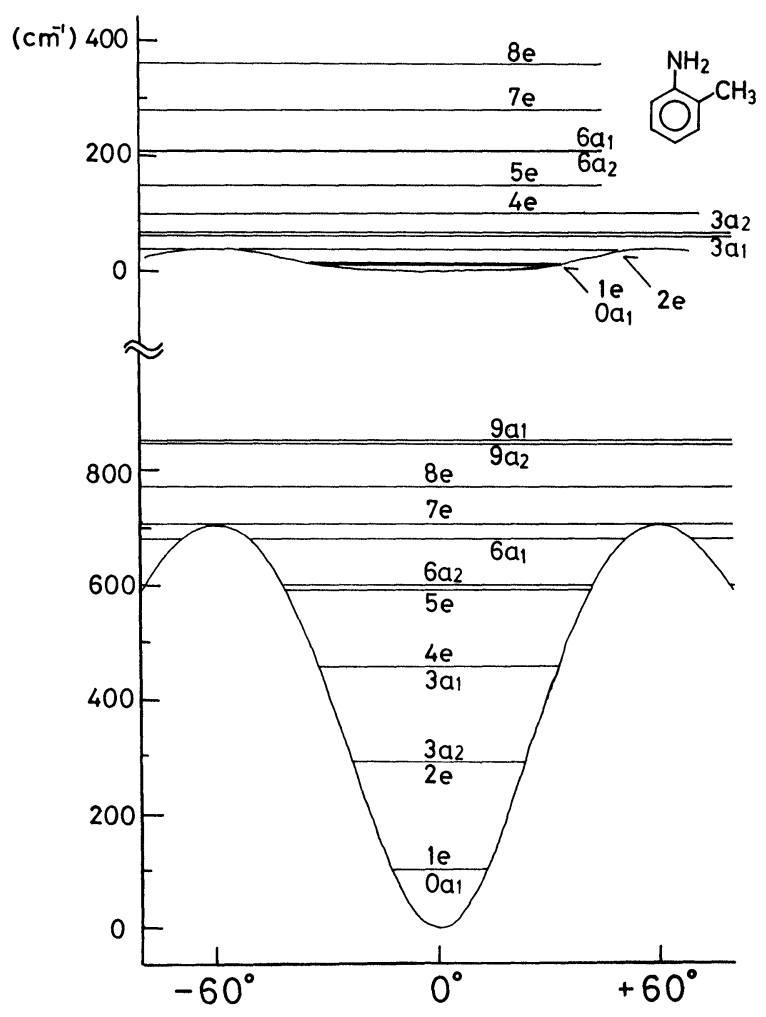

Figure 6 Potentials and energy levels for internal rotation of $o$-toluidine in the ground and excited states. 


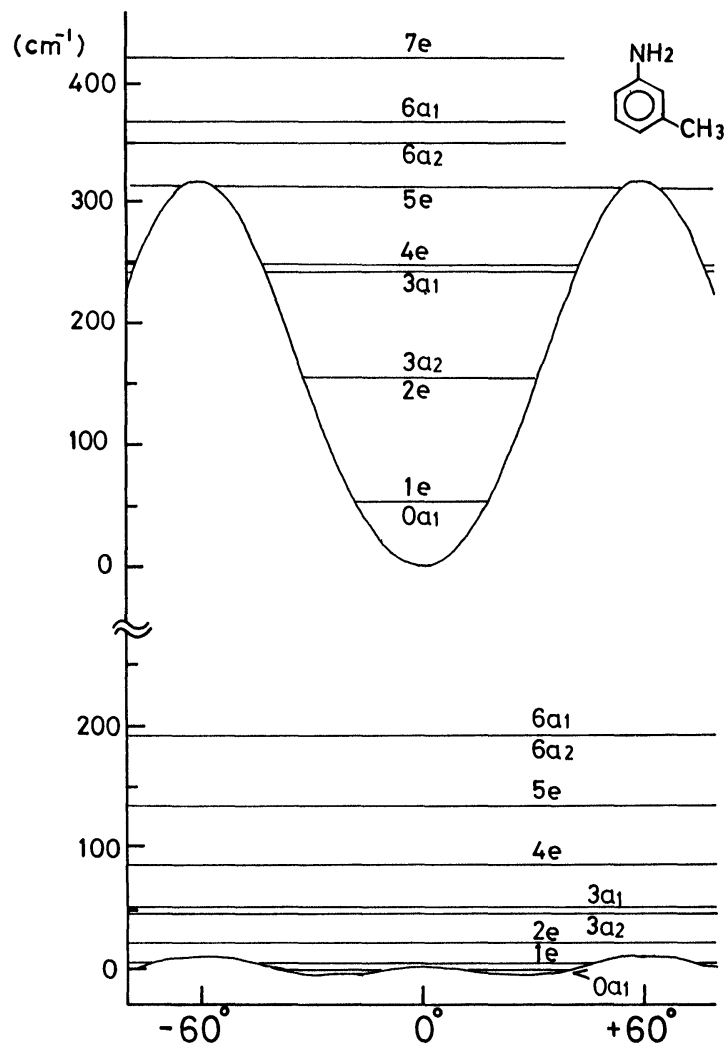

Figure 7 Potentials and energy levels for internal rotation of $m$-toluidine in the ground and excited states.

perturber. However, the internal rotation of the $\mathrm{CH}_{3}$ group is greatly affected by the local electronic and geometrical structure of the molecule in the vicinity of the $\mathrm{CH}_{3}$ group. Apparently, the large barrier height in $o$-toluidine $\left(703 \mathrm{~cm}^{-1}\right)$ arises from a great steric hindrance due to nearby $\mathrm{NH}_{2}$ group. Since $\mathrm{NH}_{2}$ is a more bulky substituent than the $\mathrm{F}$ atom, the barrier height is much larger in $o$-toluidine than in $o$-fluorotoluene $\left(288 \mathrm{~cm}^{-1}\right)$. The very small barrier height $\left(9 \mathrm{~cm}^{-1}\right)$ in $m$-toluidine is easily understood by the absence of steric hindrance, and is comparable with that of $4 \mathrm{~cm}^{-1}$ in the $S_{0}$ state of toluene $\mathrm{e}^{18}$. Therefore, the $\mathrm{NH}_{2}$ group at the $m$-position does not exert an appreciable effect on the internal rotation of the $\mathrm{CH}_{3}$ group in the $S_{0}$ state. 


\section{ABSORPTION}

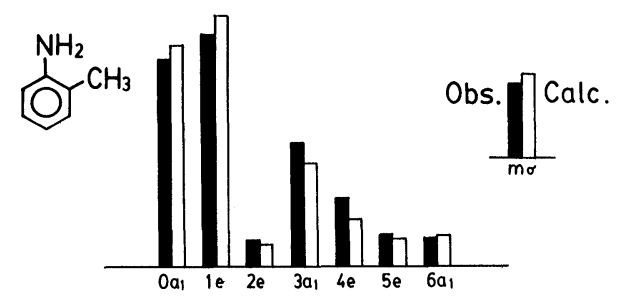

\section{EMISSION}
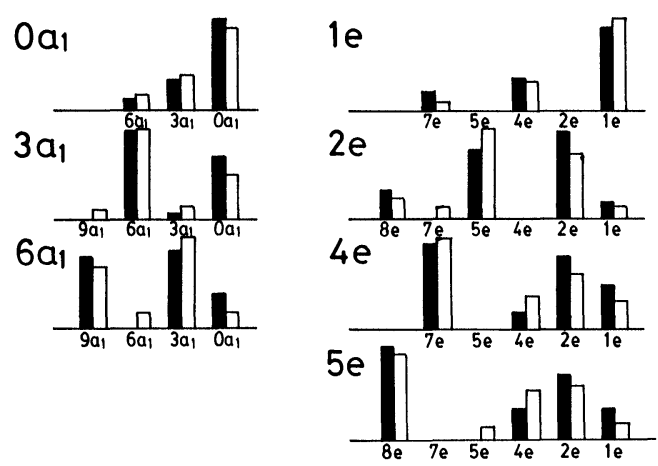

Figure 8 Comparisons between the observed and calculated intensity distributions of the fluorescence excitation and dispersed fluorescence spectra of $o$-toluidine.

Next, we shall discuss the potential barrier in the $S_{1}$ state. In the case of $m$-toluidine, the appearance of the large barrier $\left(300 \mathrm{~cm}^{-1}\right)$ in the $S_{1}$ state is difficult to explain by steric hindrance. Since the reduced rotational constant B is nearly equal betweeen the $S_{0}$ and $S_{1}$ states (see Table III), steric environment does not differ greatly between the two states. It seems reasonable that an electronic effect is mainly responsible for the appearance of the large barrier. Table IV summarizes the barrier heights of toluene, $m$-fluorotoluene, $m$-cresol and $m$-toluidine in the $S_{0}$ and $S_{1}$ states. In the table are also shown the calculated $\pi$-electron densities at $m$-carbon atoms of the parent molecules, benzene, fluorobenzene, phenol and aniline, in the $S_{0}$ and $S_{1}$ states ${ }^{19,20}$. It is seen from the table that there exists good correlation between the 

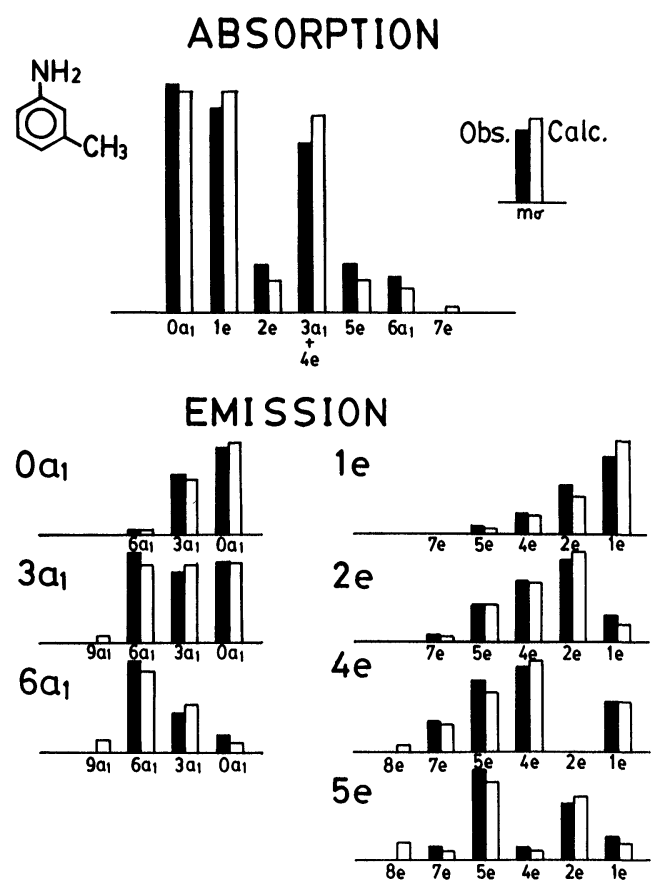

Figure 9 Comparisons between the observed and calculated intensity distributions of the fluorescence excitation and dispersed fluorescence spectra of $m$-toluidine.

Table IV Barrier heights of several $m$-substituted benzenes in $S_{0}$ and $S_{1}$ states and calculated $\pi$-electron densities of $m$-carbon atoms of parent molecules.

\begin{tabular}{|c|c|c|c|c|c|}
\hline \multirow[t]{2}{*}{ Molecule } & & \multicolumn{2}{|c|}{$S_{0}$} & \multicolumn{2}{|c|}{$S_{1}$} \\
\hline & & $\begin{array}{l}\text { Barrier } \\
\text { height } \\
\left(\mathrm{cm}^{-1}\right)\end{array}$ & $\begin{array}{l}\pi \text {-electron }{ }^{a} \\
\text { density }\end{array}$ & $\begin{array}{l}\text { Barrier } \\
\text { height } \\
\left(\mathrm{cm}^{-1}\right)\end{array}$ & $\begin{array}{l}\pi \text {-electron }{ }^{a} \\
\text { density }\end{array}$ \\
\hline Toluene & $-\mathbf{H}$ & $4^{b}$ & 1.00 & 4 & 1.00 \\
\hline$m$-Fluorotoluene & $-F$ & $17^{\mathrm{c}}$ & 1.00 & $124^{\mathrm{c}}$ & 1.04 \\
\hline$m$-Cresol & $-\mathrm{OH}$ & $10^{\mathrm{d}}$ & 1.01 & $200^{\mathrm{d}}$ & 1.07 \\
\hline$m$-Toluidine & $-\mathrm{NH}_{2}$ & 9 & 1.01 & 317 & 1.09 \\
\hline
\end{tabular}

${ }^{a} \pi$-Electron of $m$-carbon atoms of the parent molecules, benzene, fluorobenzene, phenol and aniline taken from the literature ${ }^{19,20}$.

\footnotetext{
${ }^{b}$ From Rudolph et al. ${ }^{18}$

${ }^{\mathrm{c}}$ From Okuyama et al. ${ }^{8}$

d From K. Okuyama, N. Mikami and M. Ito, unpublished data.
} 
barrier height and the $\pi$-electron density. In the $S_{0}$ state, the $\pi$-electron density at the $m$-carbon atom is nearly equal for all the parent molecules. This is reflected by the nearly free internal rotation of the $\mathrm{CH}_{3}$ group in all the $m$-substituted molecules. In the $S_{1}$ state, however, the $\pi$-electron density increases markedly in the order benzene, fluorobenzene, phenol and aniline. In the same order, the barrier height progressively increases in the $m$-substituted molecules $(4,120$, 200 and $300 \mathrm{~cm}^{-1}$ for toluene, $m$-fluorotoluene, $m$-cresol and $m$ toluidine, respectively). It is concluded therefore that the barrier to the internal rotation of the $\mathrm{CH}_{3}$ group is controlled by the $\pi$-electron density of the ring carbon atom of the parent molecule to which the $\mathrm{CH}_{3}$ group is attached. The close correlation between the barrier height and the $\pi$-electron density suggests that, in the case of the $m$-substituted molecule, the origin of the barrier to the internal rotation is the hyperconjugation of the $\mathrm{CH}_{3}$ group. The hyperconjugation contributes to the increase in double bond character of the $m$-carbon $-\mathrm{CH}_{3}$ bond depending on the electron density at the $m$-carbon atom. It is concluded that the great increase of the barrier height in the $m$-substituted molecule upon electronic excitation results from the enhancement of hyperconjugation. Recent $a b$ initio calculation of the electronic structure of $m$-fluorotoluene in the $S_{0}$ and $S_{1}$ states carried out by Nishimoto reproduces the observed potentials and supports the effect of hyperconjugation.

The dramatic decrease in the barrier height in the $S_{1}$ state of $o$-toluidine is not easy to understand. Since the situation is similar to the case of $o$-fluorotoluene, the origin of the great decrease will be the same between $o$-toluidine and $o$-fluorotoluene. In the case of $o$ fluorotoluene, we explained the almost free internal rotation in the $S_{1}$ state by a cancellation of the great steric repulsive interaction by some attractive interaction, such as intramolecular hydrogen bonding between a hydrogen atom of the methyl group and the $F$ atom. Recently, Nishimoto ${ }^{21}$ calculated the potentials for the internal rotation of the $\mathrm{CH}_{3}$ group in $o$-fluorotoluene and obtained the calculated potentials which agree very well with the observed ones. From the calculation, they concluded that electrostatic interaction between the $\mathrm{F}$ atom and the hydrogen atoms of the nearby $\mathrm{CH}_{3}$ group is responsible for the small barrier height in the $S_{1}$ state. A similar electrostatic interaction is probably a cause of the small barrier in $o$-toluidine. A possible attractive interaction would be intramolecular 
hydrogen bonding between the nitrogen atom of the $\mathrm{NH}_{2}$ group and the hydrogen atoms of the $\mathrm{CH}_{3}$ group.

In the present work, we did not report the result for $p$-toluidine. Although the fluoroscence excitation spectrum of jet-cooled $p$ toluidine was observed, the bands due to the internal rotation of the $\mathrm{CH}_{3}$ group were so weak that their detailed analysis was not possible. The weak intensity suggests that the potential for the internal rotation in $p$-toluidine does not differ greatly between the $S_{0}$ and $S_{1}$ states.

\section{References}

1. M. Baba, I. Hanazaki and U. Nagashima, J. Chem. Phys. 82, 3938 (1985).

2. A. Ron, M. Noble and Edward K. C. Lee, Chem. Phys. 83, 215 (1984).

3. D. W. Werst, W. R. Gentry and P. F. Barbara, J. Phys. Chem. 89, 729 (1985).

4. K. Yamasaki, K. Arita, O. Kajimoto and K. Hara, Chem. Phys. Lett. 123, 277 (1986).

5. C. S. Parmenter and B. M. Stone, J. Chem. Phys. 84, 4710 (1986).

6. O. Kajimoto, K. Yamasaki, K. Arita and K. Hara, Chem. Phys. Lett. 125, 184 (1986).

7. K. Okuyama, T. Hasegawa, M. Ito and N. Mikami, J. Phys. Chem. 88, 1711 (1984).

8. K. Okuyama, N. Mikami and M. Ito, J. Phys. Chem. 89, 5617 (1985).

9. K. Okuyama, T. Kakinuma, M. Fujii, N. Mikami and M. Ito, J. Phys. Chem. 90, 3984 (1986).

10. S. Kamei, K. Okuyama, H. Abe, N. Mikami and M. Ito, J. Phys. Chem. 90, 93 (1986).

11. T. Suzuki, N, Mikami and M. Ito, J. Phys. Chem. 90, 6431 (1986).

12. N. Mikami, A. Hiraya, I. Fujiwara and M. Ito, Chem. Phys. Lett. 74, 531 (1980).

13. H. Longuet-Higgins, Mol. Phys. 6, 455 (1963).

14. P. Bunker, Molecular Symmetry and Spectroscopy (Academic Press, London, 1979).

15. J. Lewis, T. Molloy, T. Chao and J. Laane, J. Mol. Struct. 12, 427 (1972).

16. M. Quack and M. Stockburger, J. Mol. Spectrosc. 43, 87 (1972).

17. R. A. Kydd and P. J. Krueger, J. Chem. Phys. 72, 280 (1980).

18. H. D. Rudolph, H. Dreizler, A. Jaeschke and P. Wendling, Z. Naturforsch. 22a, 940 (1967).

19. J. Yadav, P. Mihara and D. Rai, Mol. Phys.. 26, 193 (1973).

20. P. Mihara and D. Rai, Int. J. Quant. Chem. 6, 47 (1972).

21. K. Nishimoto, private communication. 